\title{
COMPASS results on Collins and Sivers asymmetries for charged hadrons
}

\author{
Anna Martin $¥$ \\ on behalf of the COMPASS Collaboration \\ $\ddagger$ Trieste University and INFN, Trieste, Italy
}

\begin{abstract}
The study of transverse spin and transverse momentum effects is an important part of the scientific program of COMPASS, a fixed target experiment at the CERN SPS taking data since 2002. The studies are carried on by measuring the hadrons produced in deep inelastic scattering (DIS) of $160 \mathrm{GeV} / \mathrm{c}$ muons off different targets. Among the possible asymmetries in the hadron azimuthal distributions, particularly interesting are the Collins and Sivers asymmetries which the COMPASS Collaboration has measured using transversely polarised deuteron and proton targets. Here new results for charged pions and kaons obtained from the 2010 run with a transversely polarised proton target are presented for the first time.
\end{abstract}

In the last ten years, remarkable progress has been done in the understanding of the nucleon structure, and in particular the transverse spin and the transverse momentum structure [1]. Thanks to this worldwide theoretical and experimental effort, today it is well known that in order to describe the nucleon structure in the collinear approximation, three parton distribution functions (PDFs) are needed for the different flavours $q$ : to the two quite well known unpolarised distributions $f_{1}^{q}(x)$ and the helicity distributions $g_{1}^{q}(x)$ one has to add the transversity PDFs $h_{1}^{q}(x)$, which describe the transverse polarisation of quarks inside transversely polarised nucleons. When considering parton intrinsic transverse momentum a total of eight transverse momentum dependent (TMD) PDFs are needed, the most famous of them being the Sivers function which describes the correlation between the parton transverse momentum and the transverse spin of the parent nucleon. All these new objects are either poorly known (transversity and Sivers functions) or still unknown and at present the best channel to investigate them is semiinclusive DIS (SIDIS) thanks to the coupling with chiral-odd fragmentation functions (as in the case of transversity, coped with the Collins function) and/or final state interactions. Thus in the SIDIS cross-section several modulations in different combinations of the azimuthal angles of the final state 
hadron $\left(\phi_{h}\right)$ and of the nucleon spin $\left(\phi_{S}\right)$ in the Gamma-Nucleon reference system appear, and the measurable amplitudes of some of these modulations are just the convolutions over transverse momenta of transversity or of the other TMD PDFs with the fragmentation functions. Also, when these amplitudes are measured with different targets and for different identified final state hadrons, the contributions of quarks of different flavors can be singled out.

The Collins asymmetry is the amplitude of the $\sin \Phi_{C}$ modulation $\left(\Phi_{C}=\right.$ $\phi_{h}+\phi_{S}+\pi$ is the "Collins angle") divided by the transverse nucleon polarisation and the kinematical spin transfer coefficient. It gives the convolution of transversity and the Collins fragmentation function on which independent information is coming from measurements at Belle and BaBar. Extractions of transversity from the SIDIS and $e^{+} e^{-}$data are being performed since 2005, when the first results became available.

The Sivers asymmetry is the amplitude of the $\sin \Phi_{S}$ modulation $\left(\Phi_{S}=\right.$ $\left.\phi_{h}-\phi_{S}\right)$ divided by the transverse nucleon polarisation and gives the convolution of the Sivers function and the usual fragmentation function. As for transversity, the first extractions were performed in 2005, when the first SIDIS results were produced.

The COMPASS Collaboration has measured all the transverse spin azimuthal asymmetries for protons and deuterons [2], as well as the longitudinal spin [3] and the unpolarised azimuthal asymmetries on deuteron [4]. COMPASS has also measured the transverse spin azimuthal asymmetry in hadron pair production [5]. In this contribution the most recent COMPASS results on the Collins and the Sivers asymmetries are presented.

In COMPASS SIDIS off transversely polarised targets has been measured using a $160 \mathrm{GeV} \mu^{+}$beam and a transversely polarised deuteron $\left({ }^{6} \mathrm{LiD}\right)$ target in the years 2002, 2003 and 2004. A transversely polarised proton $\left(\mathrm{NH}_{3}\right)$ target was used in 2007 and 2010. The experimental apparatus was upgraded in 2005 when the new polarised target magnet with a larger angular acceptance was installed, and the central region of the RICH detector was improved. The data analysis is very similar for all the years and the relevant cuts applied to select the "standard sample" are also the same. In order to ensure the DIS regime, only events with photon virtuality $Q^{2}>1 \mathrm{GeV}^{2}$, fractional energy of the virtual photon $0.1<y<0.9$, and mass of the hadronic final state system $W>5 \mathrm{GeV} / \mathrm{c}^{2}$ are considered. The charged hadrons are required to have at least $0.1 \mathrm{GeV} / \mathrm{c}$ transverse momentum $p_{T}^{h}$ with respect to the virtual photon direction and a fraction of the available energy $z>0.2$. No momentum cut is applied to measure the asymmetries for charged hadrons. Charged pions and 

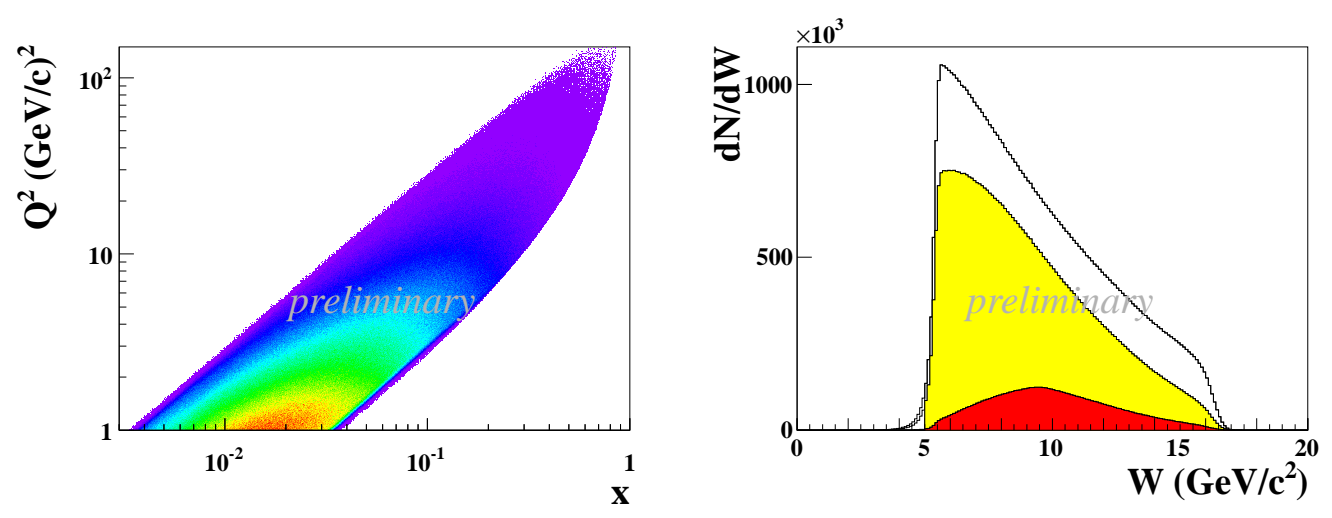

Figure 1: Left: $x-Q^{2}$ correlation for charged pions. Right: $W$ distribution for charged hadrons (upper histogram), pions (middle) and kaons (lower).

kaons are identified with the RICH detector in the momentum range from threshold $(2.7 \mathrm{GeV} / \mathrm{c}$ and $9.7 \mathrm{GeV} / \mathrm{c}$ respectively) up to $50 \mathrm{GeV} / \mathrm{c}$. The $x-Q^{2}$ correlation for charged pions from 2010 data is shown in fig. 1 (left). As can be seen, the $x$ range goes from $x \simeq 3 \cdot 10^{-3}$ to $x \simeq 0.7$ with relatively large $Q^{2}$ values in the valence region. Fig. 1 (right) gives the $W$ distribution for charged hadrons, pions and kaons. Here the different shapes are due to the quoted momentum cuts. The transverse-spin asymmetries are finally measured separately for positive and negative hadrons, pions and kaons as functions of $x, z$ or $p_{T}^{h}$ using an unbinned maximum likelihood method in which all amplitudes are fitted at the same time. The correlation between the asymmetries measured as functions of the three kinematical variables, as well as the statistical correlations between the different azimuthal asymmetries measured from the same data, have also been evaluated and, in the case of the Collins and Sivers asymmetries, they have been found to be always smaller than 0.2 [6].

The Collins and Sivers asymmetries for positive and negative hadrons on deuteron [7, 8] turned out to be compatible with zero within the few percent uncertainties, at variance with the non-zero results obtained by the HERMES experiment on proton [12, 13]. Similar results were obtained for charged pions and for kaons [9]. These data could be understood in terms of a cancellation between the $u$ and $d$ quark contributions with the deuteron target. Still, the relevance of the different $Q^{2}$ values in the overlap region $x>0.03$ of the two experiments was an open point, and a COMPASS measurement with a transversely polarised proton target was urgently needed. 


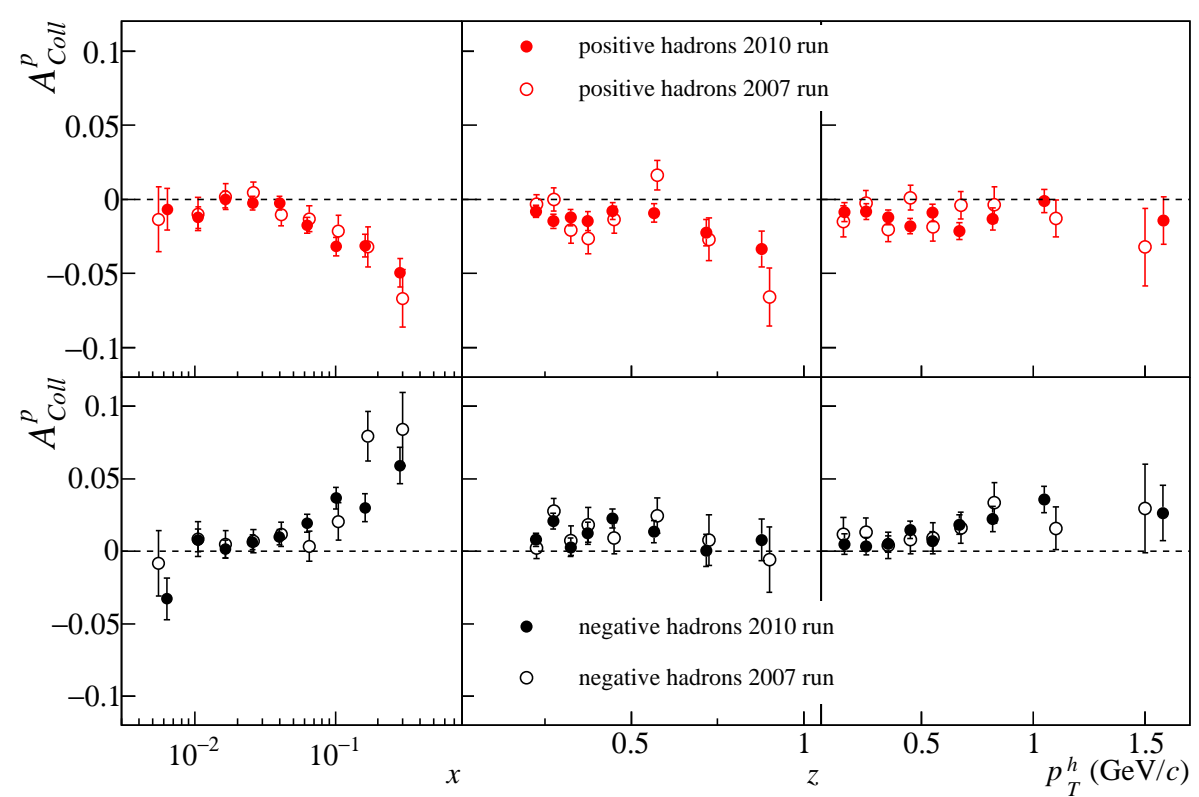

Figure 2: Collins asymmetries for positive (top) and negative (bottom) hadrons as functions of $x, z$ and $p_{T}^{h}$. The closed (open) points are the results from the 2010 (2007) data. In all the figures the error bars show the statistical uncertainties. The relevant systematic uncertainties are point to point only and they are about 0.5 the statistical uncertainties.

The first results on proton from COMPASS came from the analysis of the 2007 data [10] and gave strong evidence for non vanishing effects at COMPASS energies too. Further data with the transversely polarised proton target were taken in a longer run in 2010. The asymmetries measured from these last data [6, 11] have statistical and point-to-point systematic uncertainties about twice smaller than those of the 2007 data and their systematic scale uncertainties are negligible for all the asymmetries. The results for the Collins asymmetries for positive and negative hadrons are compared with the 2007 results in fig. 2. The same comparison for the Sivers asymmetry is shown in fig. 3. As can be seen the two independent sets of data are in very good agreement. The Collins asymmetries are compatible with zero in the previously unmeasured $x<0.03$ region while at larger $x$ they are clearly different from zero, with opposite sign for positive and negative hadrons and in nice agreement, both in sign and in magnitude, with the HERMES results [12]. The Sivers asymmetry for negative hadrons is com- 


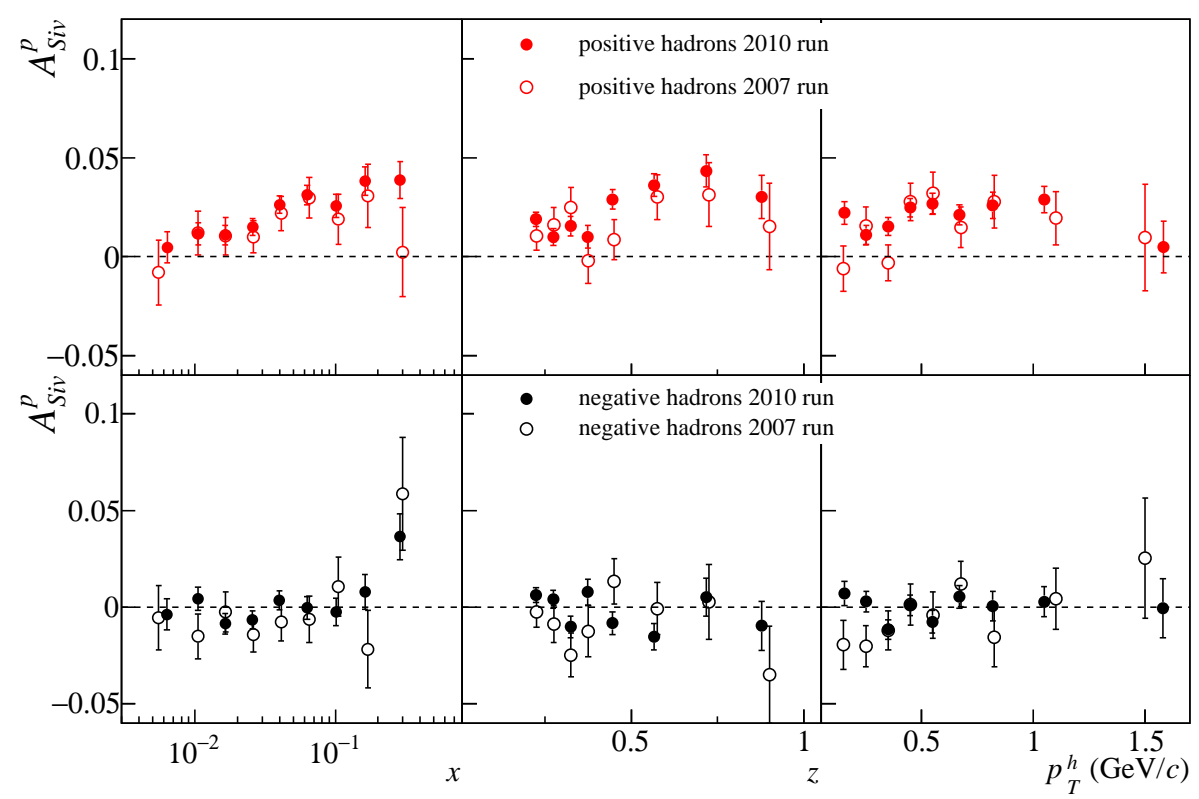

Figure 3: Same as fig. 2 for the Sivers asymmetries. For the 2007 Sivers asymmetry for positive hadrons only, an overall systematic uncertainty of 0.01 has to be added.

patible with zero with some indication for small negative values but in the last point, where both measurements give a positive value. In the case of positive hadrons, the Sivers asymmetry is positive down to very small $x$ values and smaller than the same asymmetry for pions measured by HERMES [13] in the $x>0.03$ region, a fact which can be understood in terms of the recent calculations on TMDs evolution.

The Collins and the Sivers asymmetries have also been measured for charged pions and kaons. Preliminary results from the 2007 run were produced two years ago [14]. By now the analysis of the 2010 data is also finished. As in the case of charged hadrons the preliminary results from these data are in very good agreement with the results from the 2007 data and have smaller statistical and systematic uncertainties. The Collins (Sivers) asymmetry from the 2010 data for charged pions and kaons are shown in fig. 4 (fig. 5).

As expected, the values of the pion asymmetries are very close to those of the charged hadron asymmetries, and all the previous considerations can be repeated. In particular, there is no indication for lower values of the Collins asymmetry at the higher COMPASS $Q^{2}$ values as compared to the HERMES 

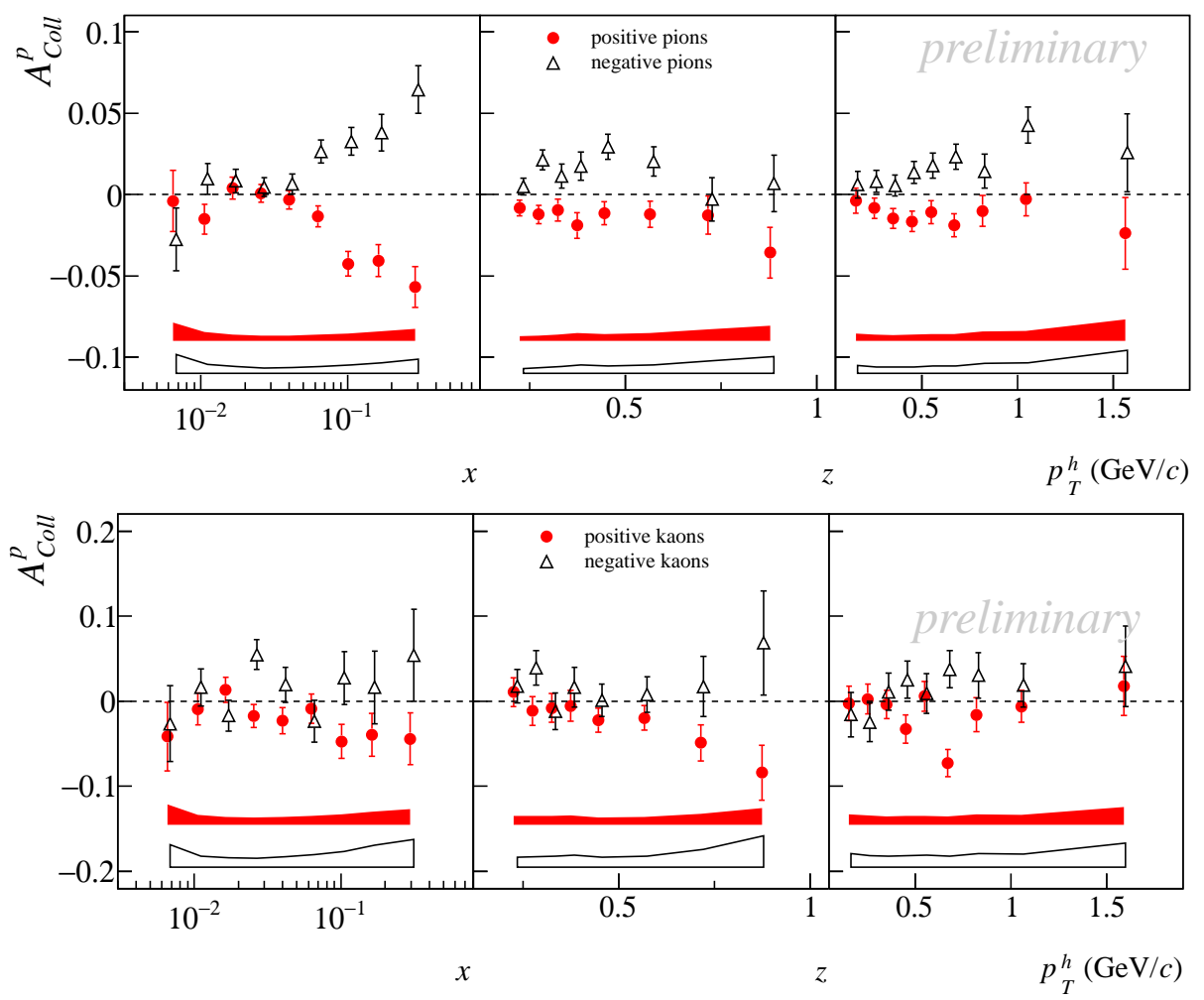

Figure 4: Collins asymmetries for positive (open points) and negative (closed) pions (upper panel) and kaons (lower panel) from the 2010 COMPASS proton data.

measurement, while the Sivers asymmetry for positive pions measured in COMPASS is definitively smaller.

For the charged kaons, the statistical uncertainties are larger, still the data give some interesting indication. For the Collins asymmetry there is some indication for different from zero values. In the case of the Sivers asymmetry for positive kaons, the values are clearly positive, again smaller than those measured by HERMES, but higher than those measured in COMPASS for positive pions. A larger Sivers asymmetry for positive kaons was already observed by HERMES and it was a puzzling point for the global fits. It will be interesting to see which impact the new COMPASS result will give to a global analysis.

Summarising, precise measurements of the Sivers and Collins asymmetries on proton have been produced by COMPASS both for charged and identified final state hadrons. While no signal was observed in the previous measure- 

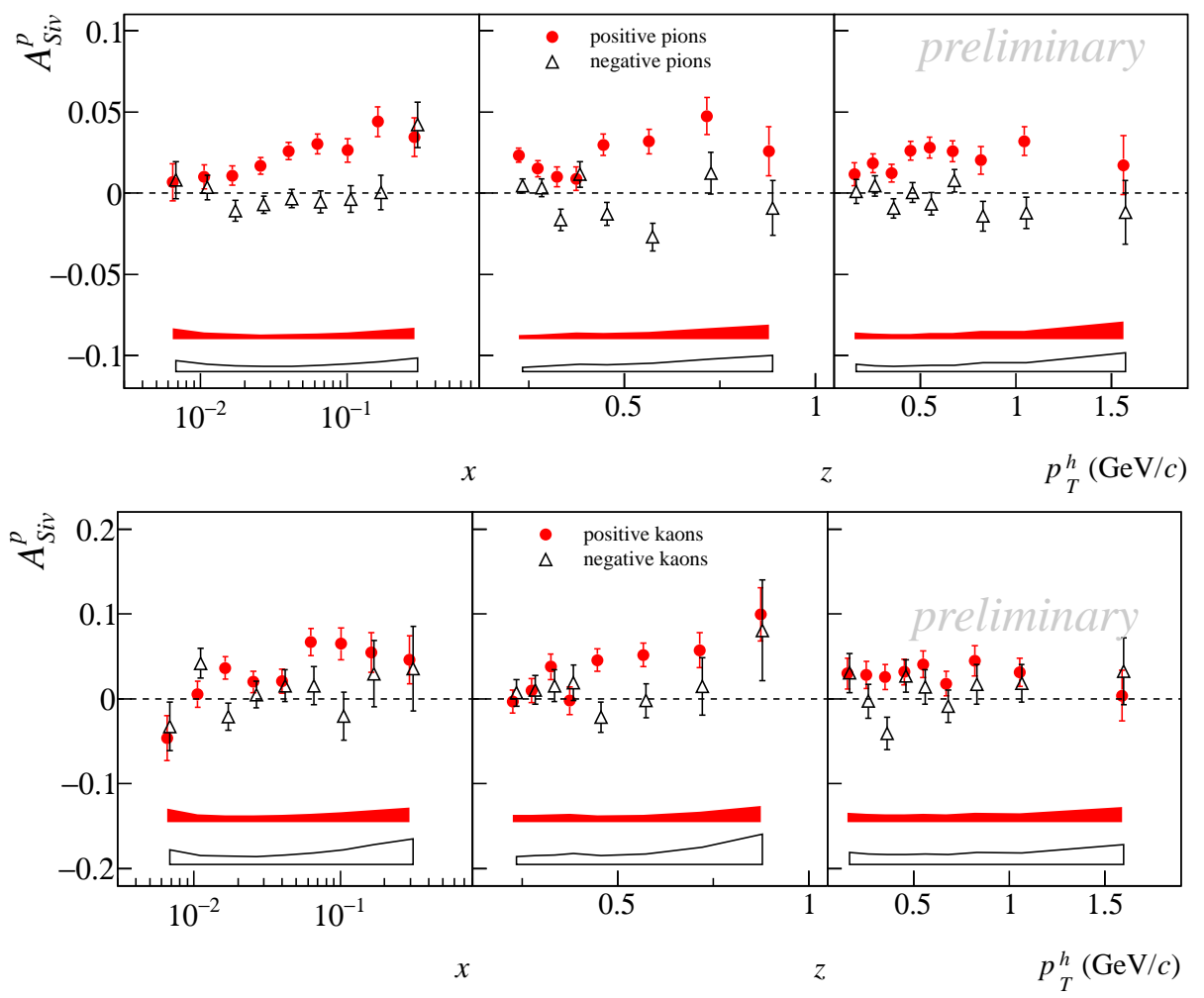

Figure 5: Sivers asymmetries for positive (open points) and negative (closed) pions (upper panel) and kaons (lower panel) from the 2010 COMPASS proton data.

ments with a deuteron target, clear non-zero signals are seen for the Collins and the Sivers asymmetries obtained from the 2007 and 2010 proton data. These data, when used together with the results at lower beam energy produced by HERMES and the past and future Jefferson Lab experiment, will give key input for testing the $Q^{2}$ evolution. More results for the Collins and Sivers asymmetries will come soon from COMPASS. In particular, thanks to the relatively high statistics of the proton data, a multidimensional analysis to better disentangle the different effects is feasible, as well as the extraction of the asymmetries in extended $z$ and $y$ ranges [6, 11]. On a longer term, the possibility to go back to the measurements with transversely polarised targets is under investigation. More precise measurements with the deuteron target are needed to better evaluate the contribution of the $d$ quark, while measurements at lower energies (e.g. $100 \mathrm{GeV}$ ) would allow to better investigate the energy dependence of the measured effects. 


\section{References}

[1] Aidala C. A., Bass S. D., Hasch D., Mallot G. K. // arXiv:1209.2803 [hep-ph];

Barone V., Bradamante F., Martin A. // Prog. Part. Nucl. Phys. 2010. V.65. P.267.

[2] Parsamian B. [COMPASS Collaboration] // these Proceedings.

[3] Alekseev M. G. et al.[COMPASS Collaboration] // Eur. Phys. J. C. 2010. V.70 P.39.

[4] Sbrizzai G. [COMPASS Collaboration] // these Proceedings.

[5] Braun C. [COMPASS Collaboration] // these Proceedings.

[6] Adolph C. et al. [COMPASS Collaboration] // Phys. Lett. B. 2012. V.717 P.376.

[7] Alexakhin V. Y. et al. [COMPASS Collaboration] // Phys. Rev. Lett. 2005. V.94 P.202002.

[8] Ageev E. S. et al. [COMPASS Collaboration] // Nucl. Phys. B. 2007. V.765 P.31.

[9] Alekseev M. et al. [COMPASS Collaboration] // Phys. Lett. B. 2009. V.673 P.127.

[10] Alekseev M. G. et al. [COMPASS Collaboration] // Phys. Lett. B. 2010. V.692 P.240.

[11] Adolph C. et al. [COMPASS Collaboration] // Phys. Lett. B. 2012. V.717 P.383.

[12] Airapetian A. et al. [HERMES Collaboration] // Phys. Lett. B. 2010. V.693 P.11.

[13] Airapetian A. et al. [HERMES Collaboration] // Phys. Rev. Lett. 2009. V.103 P.152002.

[14] Pesaro G. [COMPASS Collaboration] // J. Phys. Conf. Ser. 2011. V.295 P.012058. 